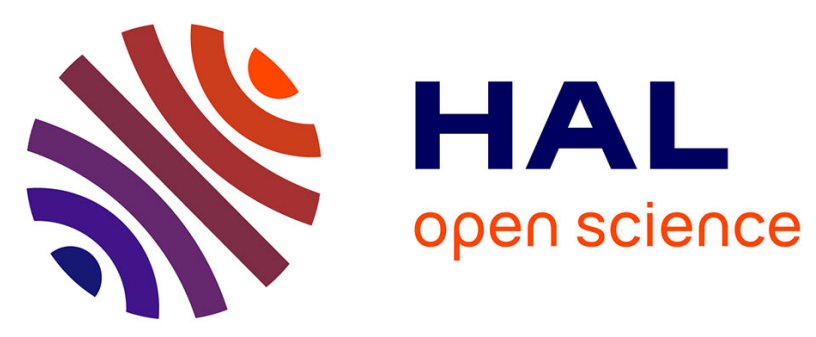

\title{
De la complexité du repérage des conduites d'explicitation dans les pratiques enseignantes de la lecture-écriture au CP
}

Anne Leclaire-Halté, Marie-José Gremmo, Maria Kreza, Natacha Espinosa

\section{To cite this version:}

Anne Leclaire-Halté, Marie-José Gremmo, Maria Kreza, Natacha Espinosa. De la complexité du repérage des conduites d'explicitation dans les pratiques enseignantes de la lecture-écriture au CP. Repères, 2017, 55, pp.205-225. 10.4000/reperes.1169 . hal-01659285

\section{HAL Id: hal-01659285 \\ https://hal.science/hal-01659285}

Submitted on 8 Dec 2017

HAL is a multi-disciplinary open access archive for the deposit and dissemination of scientific research documents, whether they are published or not. The documents may come from teaching and research institutions in France or abroad, or from public or private research centers.
L'archive ouverte pluridisciplinaire HAL, est destinée au dépôt et à la diffusion de documents scientifiques de niveau recherche, publiés ou non, émanant des établissements d'enseignement et de recherche français ou étrangers, des laboratoires publics ou privés.

\section{(ㅇ)(1) $\$$}

Distributed under a Creative Commons Attribution - NonCommercial - NoDerivatives $\mid 4.0$ 


\section{Repères}

\section{Repères}

Recherches en didactique du français langue

maternelle

Interroger l'efficacité des pratiques d'enseignement de la lecture-écriture au cours préparatoire

\section{De la complexité du repérage des conduites d'explicitation dans les pratiques enseignantes de la lecture-écriture au CP}

The identification of explicitation procedures in the teaching of reading and writing in the first year of primary school: a complex issue

Anne Leclaire-Halté, Marie-José Gremmo, Maria Kreza et Natacha Espinosa

\section{(2) OpenEdition Journals}

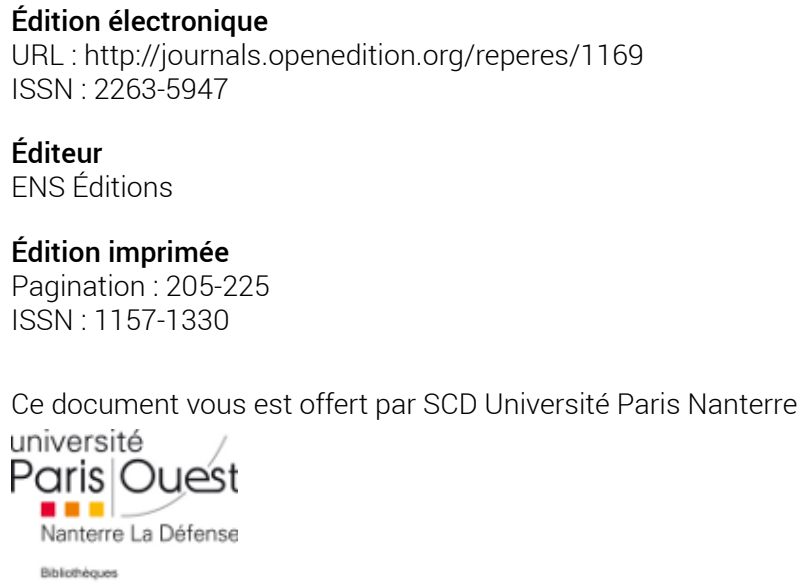

Référence électronique

Anne Leclaire-Halté, Marie-José Gremmo, Maria Kreza et Natacha Espinosa, « De la complexité du repérage des conduites d'explicitation dans les pratiques enseignantes de la lecture-écriture au CP », Repères [En ligne], 55 | 2017, mis en ligne le 22 novembre 2017, consulté le 08 décembre 2017. URL: http://journals.openedition.org/reperes/1169

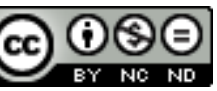

Les contenus de Repères sont disponibles selon les termes de la Licence Creative Commons Attribution - Pas d'Utilisation Commerciale - Pas de Modification 4.0 International. 


\section{De la complexité du repérage des conduites d'explicitation dans les pratiques enseignantes de la lecture-écriture au CP}

Anne Leclaire-Halté, université de Lorraine, Centre de recherche sur les médiations (CREM), Marie-José Gremmo, université de Lorraine, Laboratoire interuniversitaire des sciences de l'éducation et de la communication (LISEC), Maria Kreza, université de Crète, Laboratory of Pedagogical Research and Applications, Natacha Espinosa, université Paris-Nanterre, laboratoire Modèles, Dynamiques, Corpus (MoDyCo)

L'article se propose de revenir sur la notion d'explicitation, pour en discuter la complexité lorsqu'il s'agit d'analyser les pratiques enseignantes dans une approche écologique, comme dans la recherche Lire et écrire au CP. II propose d'abord une courte synthèse des travaux sur les fonctions de l'explicitation dans les apprentissages, fondant ainsi la légitimité de l'intérêt pour les pratiques d'explicitation des enseignants. Puis il s'appuie sur des exemples issus de l'enregistrement vidéo des séances de lecture et d'écriture dans deux classes pour se centrer sur trois des dimensions qui jouent un rôle dans l'apprentissage du lire et de l'écrire au CP : la présentation des savoirs de lecture et d'écriture, les consignes et enfin l'aide à la prise en charge du rôle d'élève. II analyse les relations que la notion d'explicitation entretient avec ces dimensions. Dans une dernière partie, il montre la variété des formes que peuvent prendre les conduites explicitantes, qui peuvent être gestuelles et/ou s'appuyer sur des artéfacts. Il pointe certaines dimensions (routinisation, style enseignant, pertinence de l'explicitation) que l'analyse a mises en évidence.

Mots-clés : apprentissage, lecture, écriture, enseignement primaire, stratégie d'apprentissage

\section{Introduction}

Un des objectifs de la recherche Étude de l'influence des pratiques d'enseignement de la lecture et de l'écriture sur la qualité des premiers apprentissages ${ }^{1}$ (Goigoux, 2016; Goigoux, Jarlégan et Piquée, 2015) a été de sonder, par des données à la fois méthodologiquement écologiques et quantitativement signifiantes ${ }^{2}$, l'hypothèse que l'explicitation joue un grand rôle dans la réussite des élèves, notamment celle des élèves en difficulté. Cette hypothèse est étayée par de

1. Lire et écrire au CP dans la suite de cet article.

2. Pour des précisions sur la méthodologie, se rapporter à l'introduction du numéro. 
nombreuses études, mais aucune de celles-ci ne porte sur l'analyse des pratiques enseignantes proprement dites. Nous avons déjà indiqué, dans un article (Crinon et al., 2015) où nous présentions les premiers éléments d'analyse des données obtenues dans l'enquête, les raisons qui ont amené notre groupe de recherche ${ }^{3}$ à s'intéresser aux conduites explicitantes des enseignants en ce qui concerne l'apprentissage de la lecture et de l'écriture au CP. Nous nous contenterons donc ici de les rappeler brièvement.

L'influence de l'explicitation sur les apprentissages de l'enfant a été montrée par des travaux issus des divers champs que sont la psychologie, la sociologie et la didactique du français. Dans le cadre de l'apprentissage de la lecture, Downing et Fijalkow (1984) ont développé le concept de clarté cognitive, qu'ils définissent comme la compréhension de deux ensembles de concepts en rapport avec la lecture et l'écriture : ceux qui concernent la compréhension des fonctions de l'écrit, et ceux, plus techniques, auxquels on a recours pour en décrire le fonctionnement. En s'intéressant plus précisément à la compréhension de la tâche de lecture par les élèves, ils accordent à la clarté cognitive une importance toute particulière. Des travaux complémentaires ont permis de préciser pour les différentes composantes de l'apprentissage de la lecture et de l'écriture, les métaconnaissances qui pouvaient être utiles (Crinon et al., 2015). Ces études mettent en avant le rôle de l'enseignant, et en particulier l'étayage qu'il propose pour amener les élèves à cette clarté cognitive : il s'agit d'expliciter ou de faire expliciter par les élèves les connaissances qui leur permettront de réaliser de nouvelles acquisitions.

Le rôle positif des pratiques d'explicitation comme levier efficace dans les apprentissages peut également être induit des travaux qui ont pointé l'existence des «malentendus sociocognitifs», entre ce que l'enseignant peut se fixer comme objectif et ce que certains élèves en perçoivent. Dans cette perspective, des travaux en sociologie de l'éducation et en didactique montrent que les modes de faire de l'enseignant peuvent aggraver ou réduire les inégalités entre les élèves (Bautier et Goigoux, 2004 ; Crinon et al., 2015 ; Rochex et Crinon, 2011). Rochex (2011) donne une importance particulière, dans la constitution ou l'aggravation des inégalités d'apprentissage, «au caractère incident ou transparent des savoirs en jeu» (p.177), lorsque les enseignants laissent à la charge des élèves de découvrir les enjeux de ces tâches et les contenus de savoir qui y sont visés.

Dans la continuité de ces études, notre hypothèse de recherche a été que la présence dans les pratiques enseignantes de conduites explicitantes jouerait un rôle dans la réussite des apprentissages, notamment pour les élèves les plus faibles et pour les moins à l'aise socialement/culturellement, en les aidant à clarifier les buts des tâches, les stratégies à mettre en œuvre, ainsi que les fonctions et le fonctionnement du langage écrit. Ainsi, les enquêteurs de Lire et écrire au CP devaient observer l'existence ou non, chez les 131 enseignants de l'échantillon,

3. Qui comprend, en plus des auteures de ce texte, Jacques Crinon (université Paris-Est Créteil, CIRCEFT) et Annette Jarlégan (université de Lorraine, LISEC). 
d'un temps d'explicitation verbale des objectifs et/ou des démarches ${ }^{4}$. La catégorie «Explicite en pourquoi» (EP) était notée lorsque l'enseignant explicitait ou faisait/laissait expliciter les finalités de la tâche (les apprentissages visés, par exemple). Une seconde catégorie «Explicite en comment » (EC) correspondait à une explicitation des procédures et des stratégies utilisables ou utilisées ou à une explicitation des connaissances mobilisées ou à mobiliser pour traiter la tâche.

Une première analyse des données obtenues sur les conduites explicitantes (Crinon et al, 2015), réalisée avec une visée descriptive, a permis de mettre en évidence la grande variabilité des pratiques enseignantes, à la fois interclasse et intraclasse, un recours très différent à ces conduites, d'une part selon le type d'explicitation relevé (EC ou EP, avec une fréquence moindre du type EP), et, d'autre part, selon les catégories de tâche concernées. Elle a également mis en évidence une diminution marquée des occurrences d'explicitation au cours de l'année. D'autre part, quand nous avons cherché à corréler pratiques d'explicitation et qualité des apprentissages, en appliquant les traitements statistiques à nos variables numériques d'occurrences d'explicitation, aucun effet significatif n'a été identifiés. Ainsi, les données empiriques recueillies sur un échantillon représentatif d'enseignants ne permettaient pas de confirmer les résultats des études menées autour de la notion de clarté cognitive.

Comment comprendre ce décalage? Nous avons cherché à répondre à cette interrogation par un travail qualitatif à partir des captations vidéo réalisées également dans le cadre de l'enquête. Nous avons sélectionné deux classes, les classes 23 et $127^{6}$, qui ont pour caractéristiques à la fois de favoriser une grande qualité des apprentissages et de proposer un grand nombre d'occurrences d'explicitation ${ }^{7}$ (cf. Goigoux, 2016). Or, l'analyse de vidéos ${ }^{8}$ a fait émerger des questions quant au repérage et à la catégorisation de conduites explicitantes chez ces deux enseignantes, et mis en évidence la complexité de la notion d'explicitation lorsqu'il s'agit d'analyser les pratiques enseignantes dans une approche écologique.

Dans les parties qui suivent, nous nous proposons de discuter de la complexité du repérage des conduites explicitantes à partir d'exemples pris dans le corpus de ces deux classes. Notre discussion se centrera sur trois des dimensions qui jouent un rôle dans l'apprentissage du lire et de l'écrire au CP, et qui ont été mises en évidence par les études citées plus haut : la présentation des savoirs de lecture et

4. Cette conduite verbale est très souvent le fait de l'enseignant, mais elle peut aussi être le fait des élèves, soit sollicitée par l'enseignant, soit produite spontanément. Nous avons estimé que l'explicitation par le maitre ou la demande d'explicitation par celui-ci procédait d'une même attention portée à l'explicitation.

5. "Les variables caractérisant le nombre de tâches donnant lieu à au moins une explicitation "en comment" ou "en pourquoi" ne produisent pas d'effet moyen sur les performances des élèves » (Goigoux, 2016, p. 412).

6. Ces numéros, attribués de manière aléatoire entre 1 et 131 , permettent d'assurer l'anonymat des classes qui ont participé à l'enquête.

7. La classe 23 est au $1^{\text {er }}$ rang en termes de nombre d'occurrences d'explicitation, et au $17^{\mathrm{e}}$ rang en termes de progression des élèves. La classe 127 est au $8^{\text {e }}$ rang en termes de nombre d'occurrences d'explicitation, et au $3^{\mathrm{e}}$ rang en termes de progression des élèves.

8. Nous avons procédé par visionnement exhaustif des vidéos concernant les deux classes, puis par transcriptions des passages présentant, selon nous, des moments d'explicitation. 
d'écriture (partie 2), les consignes (partie 3), et enfin l'aide à la prise en charge du rôle d'élève (partie 4). Dans une cinquième partie, nous nous interrogeons sur la variété des formes que peuvent prendre ces conduites explicitantes.

\section{Explicitation, savoirs, stratégies : quel repérage?}

Dans cette partie, nous avons choisi, dans l'ensemble des savoirs à présenter, de centrer notre propos sur l'explicitation des stratégies de lecture et d'écriture. En effet, le repérage de conduites explicitantes sur ces stratégies, c'est-à-dire sur comment on s'y prend pour lire et pour écrire, s'est révélé plus complexe que le repérage de conduites explicitantes autour de la conceptualisation de la nature langagière de l'écrit, qui ne fait donc pas l'objet de notre analyse ici bien qu'elle soit au centre des travaux sur la clarté cognitive cités plus haut.

La stratégie renvoie à une série d'opérations mises en place afin d'atteindre un objectif (Garner, 1990; Giasson, 1999; Romainville, 1993) et concerne une activité potentiellement consciente et contrôlable (Pressley, Forest-Pressley, Elliot-Faust et Miller, 1985; Pressley et Harris, 2008). Pressley et Harris (2008) indiquent que les stratégies sont des connaissances à propos de la manière dont on fait quelque chose, par exemple dont on décode un mot. Plus précisément, les enseignants explicitent aux élèves des stratégies pour résoudre une tâche de lecture ou d'écriture, disent ce qu'eux-mêmes sont en train de faire - en termes de stratégies - lors d'une tâche de lecture-écriture, ou font expliciter des stratégies à certains élèves, pour amener ainsi les élèves à développer une plus grande clarté cognitive à propos de la lecture et de l'écriture.

\subsection{Intrication des phénomènes}

Lorsque l'enseignant mentionne les stratégies de lecture et d'écriture, il est parfois difficile de distinguer les cas où il s'agit d'une conduite explicitante visant à rendre explicites des savoirs considérés comme implicites ou non acquis.

Ex. $1^{9}$ : cl. 127, s3 :

$\mathrm{M}$ : je vous le relis / et on va dire à quoi il faut faire attention [M lit l'extrait] / alors qu'est-ce qu'il ne faut pas oublier pendant sa copie? / A?

A : les accents

9. Pour tous les exemples cités, nous avons adopté les normes de transcription suivantes:

- pas de marques de ponctuation sauf le? pour l'intonation montante;

- «/» pour indiquer les pauses;

- l'enseignant est noté $M$;

- anonymat des élèves par l'emploi de E, EEE quand il y en a plusieurs, et l'initiale du prénom quand l'élève est nommé;

- les indications gestuelles ou posturales apparaissent entre des crochets [ ];

- les phonèmes sont transcrits selon la norme API, les graphèmes apparaissent entre «»;

- les exemples ont été parfois raccourcis pour en simplifier la lecture, et les coupures sont signalées par [...];

$-[\mathrm{xxx}]$ pour les passages inaudibles;

- utilisation de majuscules pour marquer les endroits où l'enseignante élève la voix.

Les semaines d'observation sont indiquées comme suit : s1 renvoie à la semaine de novembre 2013, s2 à celle de mars 2014 et s3 à celle de mai 2014. 
$\mathrm{M}$ : alors les accents/quoi d'autre? / les?

E : les virgules

$\mathrm{M}$ : les virgules

$\mathrm{E}$ : les points

$\mathrm{M}$ : les points/A

A : les trois points et

M : là il n'y en a pas

E : les majuscules

$\mathrm{M}$ : les majuscules/le premier mot que vous avez à écrire c'est «Julien» et on met une majuscule à «Julien» / vous faites attention. [...] T/ tu fais attention à la lettre «d» / je vais mettre un petit pense-bête au tableau/regarde / ça arrive qu'il n'y ait pas que T/vous pouvez lever la tête/regardez [M écrit au tableau] / donc ça c'est le « $\mathrm{d}$ » en script/comme il est écrit sur votre livre/le voilà en attaché/alors faites attention à ne pas le confondre avec le «b» / voilà / vous pouvez regarder le tableau

E : moi parfois je confonds

$\mathrm{M}$ : je sais bien/c'est pour ça que je rappelle ça

L'enseignante fait expliciter les éléments de savoir à prendre en compte pour copier correctement un passage. Elle rappelle, à la fin du passage, la différence, au niveau de la forme écrite, entre la lettre «b» et la lettre « $d$ ». Elle souligne que, lors de la copie, les élèves peuvent s'aider de ce qu'elle a écrit au tableau.

Étant donné que nous sommes au dernier trimestre et que les élèves ont déjà fait des exercices de copie et sans doute déjà écrit les lettres «b» et «d» plusieurs fois, ces verbalisations de la part de l'enseignante nous paraissent viser deux dimensions. D'une part, l'enseignante semble considérer qu'elle doit de nouveau expliciter ces savoirs (les stratégies de copie), car ils ne sont pas encore acquis par tous les élèves. D'autre part, elle explicite également certains aspects de la procédure de travail, et les aides qu'elle fournit (le pense-bête au tableau) pour réaliser la tâche. Dans cet exemple, explicitation d'une stratégie (notre EC) et présentation (ou rappel) des savoirs sont enchevêtrées, et déterminer les limites entre l'une et l'autre dans le discours de l'enseignante semble complexe.

Pour l'écriture des mots, les enseignants de notre corpus se réfèrent aux gestes à faire afin de former les lettres. Par exemple :

Ex. 2 : cl. 23, s1 :

$\mathrm{M}$ : attention la lettre «b»/ en cursive/à chaque fois vous me faites la lettre «b» en script/ / la lettre «b», si on écrit en cursive, les enfants, je n'écris pas la lettre «b» en script/la lettre «b» en cursive c'est comme la grande boucle du «1» $[. .$. mais pour pas que je la confonde avec la lettre «l» je remonte et je lui fais la petite boucle en haut [M écrit au tableau] / elle est difficile hein la lettre «b» / à chaque fois vous tombez dans le piège 
Là encore, s'agit-il de la présentation de savoirs ou peut-on parler d'explicitation? L'enseignante semble considérer comme nécessaires une explicitation des gestes d'écriture cursive, et la justification de cette explicitation (par ex. «à chaque fois vous tombez dans le piège»). On peut aussi supposer qu'elle veut présenter à nouveau aux élèves des savoirs qui ne semblent pas encore être acquis (on est au premier trimestre). De nouveau, la frontière entre explicitation et énoncé de savoir est ténue.

\subsection{Explicitation des stratégies et correction}

Nous avons constaté que dans les deux classes observées, la différence entre explicitation et correction était également problématique. En effet, les deux enseignantes ont souvent recours à l'explicitation de stratégies lorsqu'elles reprennent les élèves.

Ex. $3: \mathrm{cl} .23, \mathrm{~s} 1$

A : une fleur dans le chemin

$\mathrm{M}$ : non/là tu sais ce que tu fais? / A/tes yeux ils regardent pas les mots / tu te rappelles l'histoire que tu m'as lue tout à l'heure/la petite phrase/mais tu t'es trompé dans les mots / lire c'est pas redire une histoire hein [...] c'est déchiffrer toutes les syllabes et tous les mots / alors les yeux doivent apporter l'information dans la tête et la bouche doit dire toutes les syllabes que la tête a découvertes / on y va

Y a-t-il explicitation, correction, ou les deux à la fois? Dans un premier temps, l'enseignante explicite la stratégie erronée qu'utilise l'élève, puis elle précise la stratégie à mettre en œuvre pour une lecture correcte. Cette intervention semble avoir plusieurs fonctions : invalider la proposition de l'élève, mais aussi l'amener à prendre conscience de la différence entre ce qu'il fait et ce qu'il devrait faire. Ce faisant, elle explicite aussi la «bonne»stratégie à toute la classe. Ici, c'est la distinction entre correction et explicitation qui peut poser problème.

\section{Lors des consignes, expliciter ou expliquer?}

Une deuxième catégorie d'action enseignante est très présente dans les études sur la clarté cognitive en classe (cf. Bautier et Goigoux, 2004; Crinon et al., 2015 ; Rochex et Crinon, 2011) : il s'agit des moments où l'enseignant fournit les consignes à suivre pour la réalisation d'une tâche scolaire de lecture ou d'écriture.

Pour notre définition de la consigne, nous nous situons dans une perspective didactique : nous entendons, par ce terme, l'indication, par l'enseignant, d'une tâche ou d'un exercice de lecture-écriture aux élèves, de manière que ces derniers entrent dans cette tâche, réalisent cet exercice.

La tâche peut être donnée par écrit, avec une consigne formulée ou par l'enseignant ou par autrui (par l'auteur d'un fichier d'exercices ou d'un manuel par exemple). Elle doit alors être lue et comprise par chaque élève. Elle peut aussi être donnée à l'oral, et, dans ce cas, c'est l'enseignant qui la donne. Il se peut, 
enfin, qu'elle soit mixte (Zakhartchouk, 2000) : l'enseignant lit une consigne écrite et la reformule pour les élèves.

Pour réaliser l'apprentissage visé par la tâche, il est aussi nécessaire que les élèves comprennent les enjeux de cette tâche, qu'ils en perçoivent la finalité pour leurs apprentissages (Zerbato-Poudou, 2001). Les membres d'ESCOL ${ }^{10}$ (par ex. Bautier et Rayou, 2009) ont montré que si les élèves ne perçoivent pas la finalité de la tâche, si cette finalité n'est pas verbalisée, en particulier pour ceux d'entre eux qui, de par leur appartenance aux classes populaires, ne sont pas familiarisés avec les enjeux de l'école, alors ils resteront au simple niveau du «faire», et apprendront peu.

L'explicitation intervient, dans la consigne, quand cette dernière est accompagnée de l'indication de stratégies à utiliser pour réaliser la tâche : on a alors affaire à une explicitation en comment. Elle peut également intervenir quand ce sont les objectifs visés par la tâche qui sont donnés : il s'agit ici d'explicitation en pourquoi.

C'est pourquoi la consigne, qui fait partie intégrante de la tâche, est un lieu d'explicitation en comment et en pourquoi non négligeable. Cependant, cette explicitation, considérée comme une expansion possible de la consigne qui s'attache à l'enjeu de la tâche et/ou à la manière de la réaliser, peut être difficile à distinguer de l'explication, qui reformule, parfois en le développant, le premier énoncé de la tâche. Nous allons en discuter en distinguant les explicitations orales formulées à partir d'une consigne écrite, des explicitations données dans une consigne fournie uniquement oralement.

\subsection{Explicitations orales à partir d'une consigne écrite}

Les consignes écrites recèlent une part d'implicite (Zakhartchouk, 2000), que tous les élèves ne sont pas en mesure de comprendre. De plus, au CP, en particulier parce que les élèves ne sont pas toujours en mesure de lire intégralement une consigne, l'enseignant la reformule souvent oralement, alors qu'elle est écrite sur le cahier, sur le manuel ou le fichier d'exercices, comme le montrent par exemple les observations faites par Leclaire-Halté (2007) sur les pratiques ordinaires d'une enseignante de CP et par Bishop et Sraïki (2007) sur celles d'enseignants dans un dispositif de CP renforcé.

Ex. 4 : cl. 127 , s3

$\mathrm{M}$ : écris la phrase en remplaçant les mots en gras par les mots qui conviennent/les mots en gras sont des mots qui sont écrits de quelle manière? P?

$P$ : en gros et en noir

$\mathrm{M}$ : voilà / en gros et en noir/combien y a-t-il des mots en gras? / D

$\mathrm{D}:$ deux

10. Éducation et scolarisation, équipe du CIRCEFT (Centre interdisciplinaire de recherche «culture, éducation, formation, travail»), un laboratoire de recherche en éducation des universités Paris 8 Vincennes-SaintDenis (établissement principal) et Paris-Est Créteil (établissement secondaire). 
M : il y a deux mots à transformer/d'accord? / là je ne lis pas la phrase/lisez bien les mots en gras/dans chaque mot il n'y a qu'une seule lettre à modifier/à changer/d'accord? /ça va ou pas S?

$S:$ j'ai pas compris

$\mathrm{M}$ : alors qui peut réexpliquer pour $\mathrm{S}$ ? M?

M. : $[\mathrm{xxx}]$

M : oui / mais quel est son problème? / les mots en gras quel est leur problème? / pourquoi ça ne nous convient pas?

$\mathrm{E}$ : parce qu'ils sont pas bien écrits $[\mathrm{xxx}]$ avec la phrase

$\mathrm{M}$ : voilà / la phrase n'a aucun sens à cause des mots écrits en gras / pour que la phrase ait un sens/pour qu'elle raconte quelque chose d'intéressant/il faut les transformer ces mots qui sont écrits en gras / d'accord? il faut enlever une lettre/ et mettre une autre à la place pour que la phrase veuille dire quelque chose

Dans cet exemple, l'enseignante lit la consigne écrite. Puis elle s'assure que les élèves ont compris ce qui leur est demandé. Tout d'abord, elle explique le sens technique du mot «gras». Cette explication est au service de l'explicitation d'une stratégie : il faut remplacer ce mot en gras par un autre mot qui ne se différencie du précédent que par une lettre. Un obstacle cognitif est manifesté par l'élève $\mathrm{S}$, ce qui conduit l'enseignante à préciser une dimension supplémentaire de l'exercice : l'opération doit permettre d'obtenir une phrase au sens satisfaisant. Explique-t-elle la consigne ou l'explicite-t-elle? Il n'est pas facile de différencier, ici, l'explication de l'explicitation. En effet, l'enseignante explique la consigne et donne des stratégies pour réaliser l'exercice : repérer les deux mots à modifier, ne changer qu'une lettre de ces derniers, vérifier que la phrase obtenue a un sens... Les deux opérations semblent fortement intriquées.

La différence que nous faisons entre explication et explicitation apparait plus nettement dans l'exemple suivant :

Ex. 5 : cl. 23, s3

$\mathrm{M}$ : cette consigne elle est un peu compliquée/croissant oui c'est ça hein/elle est difficile cette consigne [...] alors attends s'il te plait /alors B il nous dit/la consigne est difficile/parce que c'est dans l'ordre croissant qui est difficile/ mais ça je vais vous l'expliquer/mais B il nous dit la consigne est difficile/mais l'exercice il est pas du tout difficile/c'est tant mieux ça pour nous parce que/retenez bien/ordre croissant/du plus petit au plus grand/ça vous allez le trouver dans tous les exercices de mathématiques/et hier on a vu le mot collégien/vous avez tous des frères et des sœurs au collège/ je suis sure que dans leurs exercices de mathématiques il y a le mot «ordre croissant»

L'enseignante explique l'expression «dans l'ordre croissant» : «du plus petit au plus grand». Mais elle est dans l'explicitation quand elle rappelle pourquoi c'est important de savoir ce que signifie l'expression et de la mémoriser, c'està-dire les enjeux de la bonne connaissance de l'expression : c'est que les élèves la rencontreront, au cours de leur scolarité, dans de nombreux problèmes et 
exercices. Connaitre cette expression contribuera ainsi, d'une certaine manière, à leur réussite scolaire, leur permettra de comprendre de nombreuses consignes et énoncés. Ici, la différence entre explicitation et explication apparait plus nettement. Le sens de l'expression « de l'ordre croissant» est donné, cette expression est expliquée aux élèves. Quand il est question de l'importance de connaitre cette expression, on a bien une explicitation en pourquoi. Cette différence est moins perceptible dans l'exemple 4, où, en expliquant, l'enseignante explicite en même temps des stratégies à mettre en place pour réaliser correctement l'exercice (explicitation en comment).

Les deux exemples suivants montrent ainsi qu'il est plus délicat, dans une consigne, d'identifier comme une explicitation ce qui est une mise en avant de stratégies (notre EC) que ce qui est une mise en avant d'enjeux (notre EP).

Ex. 6 : cl. 127 , s1

$\mathrm{M}$ : écris « $\mathrm{t}$ » quand tu entends [t] / si on n'entend pas [t] qu'est-ce qu'on fait?

EEE : on n'écrit pas

$\mathrm{M}$ : on n'écrit rien/alors on ouvre grand ses oreilles / où est-ce qu'on va écrire la lettre «t» si on entend le son?

EEE : sur le trait bleu

$\mathrm{M}$ : sur le trait bleu

Ex. 7 : cl. 23, s2

$\mathrm{M}$ : à ton avis pourquoi est-ce que j'ai fait un exercice avec des «on» et un exercice avec des «ou»? [...] c'est pour entrainer vos yeux à bien voir que le «on» $\mathrm{c}^{\prime}$ est le «o » avec le «n» / mais quand on écrit en script/le «n» ressemble au « $\mathrm{u} »[\ldots] /$ mais ils ne font pas du tout le même son

Dans l'exemple 6, l'enseignante lit la consigne, qu'elle explique et explicite en même temps, puisqu'elle donne des stratégies pour faire l'exercice. Dans l'exemple 7, les élèves découvrent la fiche des devoirs à faire à la maison. Un élève remarque que dans l'exercice 1 , il y a des «on» et des «ou». L'enseignante explicite alors pourquoi elle a élaboré l'exercice, ce qu'il va permettre de travailler chez les élèves. L'exemple 7 met en avant les enjeux d'apprentissage de l'exercice, pourquoi l'enseignante a donné cet exercice : ce dernier est conçu comme un entrainement par rapport à une difficulté que peuvent rencontrer les élèves, la confusion entre les deux graphies «ou » et «on » en caractères scripts.

\subsection{Explicitations dans les consignes orales}

Il s'agit des consignes qui sont données oralement par l'enseignant sans support écrit. Les consignes orales peuvent être plus ou moins ouvertes, aller de l'annonce de ce qui va faire l'objet de la tâche à la précision des modalités de l'accomplissement de cette tâche. Parfois, les consignes données oralement ne posent pas de problème quant au repérage de la part qu'elles laissent à l'explicitation, comme au début de l'exemple 8. 
Ex. 8 : cl. 127, s3

$\mathrm{M}$ : on va travailler sur la phrase/on va faire une phrase puzzle

$E$ : ah j'adore ça

$\mathrm{M}:[\ldots]$ explique la consigne

$\mathrm{E}:$ c'est une phrase/on met dans l'ordre

$\mathrm{M}$ : on met dans l'ordre quoi?

$\mathrm{E}:$ des mots

M : des mots / donc je vais vous écrire des mots dans le désordre au tableau / et vous allez essayer de les remettre dans l'ordre pour constituer une?

EEE : phrase

$\mathrm{M}$ : qu'est-ce qui va nous aider? / quels indices on va prendre?

$\mathrm{E}$ : la majuscule et le point

$\mathrm{M}$ : d'accord/le mot qui aura une majuscule on va le mettre au

EEE : début

$\mathrm{M}$ : au début de la phrase/le mot qui sera suivi d'un point sera le?

EEE : dernier

$\mathrm{M}$ : le dernier

Dans un premier temps, l'enseignante donne l'objectif de la tâche : «on va travailler sur la phrase». Même si cette explicitation en pourquoi est partielle, elle peut permettre à des élèves qui voudraient raconter ce qu'ils ont fait en classe à leurs parents, de dire autre chose que «j'ai copié des mots au tableau», ce qui témoignerait de leur maintien dans le «faire». Puis l'enseignante annonce la nature de la tâche elle-même, la phrase puzzle, tâche que les élèves ont déjà réalisée (cf. la première réaction de l'un d'entre eux). Enfin, comme les élèves ont déjà fait ce genre d'exercice, ils peuvent donc participer à l'explicitation des stratégies à mettre en place pour la tâche. Ils expliquent comment accomplir cette dernière, tout en explicitant ses différentes phases, et, pour nous, cette explicitation fait partie de la consigne.

D'autres exemples sont plus compliqués à analyser. Dans les cas de consignes orales «ouvertes», où l'enseignante annonce ce qui va composer le programme de la suite du travail, quelles sont celles où l'on peut relever de l'explicitation? L'exemple qui suit témoigne bien d'une conduite explicitante :

Ex. 9 : cl. 127, s3

M : alors / ça va être un petit peu particulier/parce que je vous ai préparé l'écriture mais ce n'est pas par ça que nous allons commencer/ce matin on va faire une petite dictée de syllabes / pour le travail de lecture que l'on fait d'habitude sur l'ardoise / ça sera une dictée de syllabes dans le cahier pour voir un peu où vous en êtes/au niveau des sons / syllabes 
L'enseignante annonce la tâche aux élèves en même temps qu'elle donne son objectif pédagogique, qui est l'évaluation du degré d'acquisition des élèves des savoirs en cours. Nous sommes donc en présence d'une explicitation en pourquoi. Par contre, un énoncé plus bref, du type «maintenant on va étudier les façons d'écrire [s] », s'il annonce la tâche à venir aux élèves, est beaucoup moins précis quant à l'objectif pédagogique : il est plus difficile d'y repérer une volonté d'explicitation de la part de l'enseignante.

D'autres problèmes liés au repérage de l'explicitation peuvent surgir face au type d'échange, entre l'enseignante et les élèves, de l'exemple 10 :

Ex. $10:$ cl. 127, s2

$\mathrm{M}$ : les consignes avant/j'ai oublié de les rappeler/qu'est-ce qu'on fait? [...] R

$\mathrm{R}$ : on lit fort

$\mathrm{M}$ : on essaie de bien lire fort $[\ldots]$ / C

$\mathrm{C}:[\mathrm{xxx}]$

$\mathrm{M}$ : et on essaie de bien mettre le ton/D

$\mathrm{D}:[\mathrm{xxx}]$

$\mathrm{M}$ : on suit avec son doigt pour ne pas être perdu / et quand il y a un point qu'est-ce qu'on fait?

E : on s'arrête

$\mathrm{M}$ : on essaie de lire/vous commencez à lire plus fort/on y est presque

Il s'agit ici de lire à haute voix. Mais que penser de ce passage où l'enseignante se livre à un rappel des conditions de la lecture à haute voix? Est-ce le «simple» énoncé de consignes correspondant à la tâche «lecture à haute voix »? Est-ce l'explicitation des stratégies à employer, plus précisément un rappel d'explicitation que l'enseignante construit avec certains élèves, pour que tous soient au clair sur ce que la tâche implique?

\section{Expliciter, organiser, faire de la discipline?}

Dans cette partie, nous nous intéresserons plus globalement aux liens entre activités de lecture et d'écriture, et rôle d'élève. Plutôt que de "métier d'élève», nous choisissons ici de parler de «rôle d'élève» pour nous centrer sur le rôle social que l'obligation scolaire demande aux enfants d'assumer à l'école ${ }^{11}$. Comme nous l'avons rappelé dans la première partie, la sociologie de l'éducation a montré combien la compréhension de la nature du rôle d'élève, et sa prise en charge comportementale, était gage, pour chaque enfant, de la meilleure réalisation possible des apprentissages scolaires. Elle a aussi montré la distance qui peut

11. Dans les écrits des auteurs qui travaillent la notion de «métier d'élève», les termes de «métier» et de «rôle» sont souvent interchangeables : cf. Perrenoud (1996, n. p.) : «Être élève [...] c'est un statut, une condition, caractérisée par l'exercice d'un rôle spécifique, que j'ai considéré comme un "métier". » 
exister, pour un certain nombre d'enfants, entre le rôle d'enfant que leur famille leur propose de prendre en charge, et le rôle d'élève que l'école exige d'eux. Ainsi, certains d'entre eux, «faute de modèle par rapport auquel se situer, reproduisent des comportements existants dans d'autres espaces sociaux» (Meirieu, n. d., p.2). L'entrée au CP, si elle se fait pour la très grande majorité des enfants dans une certaine continuité avec leur expérience de l'école maternelle, représente néanmoins pour eux une nouvelle rupture, et implique notamment qu'ils comprennent et acceptent une nouvelle situation scolaire. Une partie du rôle de l'enseignant est donc d'aider les enfants à entrer dans leur rôle d' «élève de primaire», qui va leur permettre de donner un sens au travail scolaire (Perrenoud, 1994). La clarté cognitive sur les apprentissages inclut la clarté cognitive sur l'ensemble de la situation scolaire, et notamment sur le rôle d'élève. Si ce concept s'appliquait d'abord, dans les études citées plus haut, aux activités de lecture et d'écriture au sens strict, nous l'appliquons à l'ensemble de la situation scolaire, et notamment à la compréhension du rôle d'élève.

Lorsque, dans l'analyse des enregistrements vidéo, nous avons repéré des conduites explicitantes de l'enseignant vis-à-vis de la socialisation au rôle d'élève, il ne nous a pas toujours été facile de distinguer explicitation des rôles sociaux, présentation des savoirs, organisation scolaire et discipline : l'objectif de socialisation au rôle d'élève, difficilement dissociable de la fonction d'instruction, se trouve souvent intégré dans les activités d'enseignement-apprentissage proprement dites.

\subsection{Travailler les savoirs, expliciter le rôle d'élève}

L'exemple suivant témoigne de la manière dont apprentissage des savoirs scolaires et prise en charge du rôle d'élève peuvent être fortement intégrés :

Ex. 11 : cl. 23, s1

L'enseignante a demandé aux élèves de fournir des mots où l'on entend le son [R]

$\mathrm{M}$ : oui D? Ah D qu'est-ce que tu nous montres? [M se tourne vers le fond de la classe] mais qu'est-ce que c'est que cette fiche?

$\mathrm{N}:[\mathrm{xxx}]$ les choses qu'il faut faire

$\mathrm{M}$ : exactement merci N/mais comment est-ce qu'on avait appelé ce travail? ah/je crois que j'ai entendu quelqu'un / T

$\mathrm{T}$ : les règles de la classe

$\mathrm{M}$ : eh ben merci T [M prend son bâton et va au fond de la classe] / le petit article «les» et regardez/qu'est-ce que j'ai fait là?

$\mathrm{E}:$ un $« \mathrm{~s} »$ muet

$\mathrm{M}: \mathrm{le} « \mathrm{~s} »$ muet

$\mathrm{E}$ : parce qu'il y en a plusieurs

$\mathrm{M}$ : parce qu'il y en a plusieurs/il y en a plein des règles/les [R]règles [M accentue le $[\mathrm{R}]$ de règle]/ en plus regardez/ah quelle est cette lettre? 
$\mathrm{E}:$ un $« \mathrm{r} »$.

$\mathrm{M}$ : un « $\mathrm{r} » .[\mathrm{R}] /$ les / [R]règles [M accentue le $[\mathrm{R}]$ de règle $]$ de/la / classe/donc à bon entendeur salut/nous les avons fabriquées ensemble ces règles / elles sont affichées / si jamais je ne me rappelle plus qu'il ne faut pas que je dise des gros mots / je tourne la tête je regarde ici/si jamais je sais plus qu'il ne faut pas faire de travail de petit cochon je retourne la tête je regarde ici/et si jamais je ne respecte pas les règles de la classe que se passe-t-il? [...] vas-y $\mathrm{C}$

$\mathrm{C}$ : en zone rouge

$\mathrm{M}$ : oh avant de passer en zone rouge/on passe en zone? [...]

$\mathrm{M}$ et EEE : jaune/orange / rouge

Lors de l'exercice de code, un élève fait référence à la fiche des règles de la classe, et l'enseignante travaille alors à deux niveaux : elle fait relire le titre et préciser le son recherché, puis elle explicite également des aspects de la socialisation scolaire, en rappelant les règles qui constituent le rôle d'élève, en pointant la fiche où ces règles sont accessibles au fond de la classe.

De même dans l'exemple 12, on constate encore une forte intégration entre l'apprentissage de l'écrit et la socialisation dans le rôle d'élève :

Ex. 12 : cl. 23, s1 :

$\mathrm{M}$ : j'aimerais que L nous présente son travail/c'est un petit travail pour la classe/est-ce que tu peux expliquer ce petit travail L?

$\mathrm{L}:$ je/suis / un/élève

M : ça c'est ce que tu as écrit qu'est-ce qu'on va faire de cet écrit de cette petite phrase?

L : on va la coller sur la porte

$\mathrm{M}$ : on va la coller sur la porte /à l'extérieur/parce que/quand on franchira la porte d'entrée de notre classe / on lira bonjour/on lira qu'on est en classe de CP/mais on lira aussi / je suis un/élève /

E : sérieux

$\mathrm{M}$ : c'est vrai qu'on aurait pu rajouter sérieux on aurait pu rajouter?

E : concentré

E : appliqué

E : sage

E : gentil

M : gentil/oh là vous avez plein d'idées/donc on va laisser la feuille comme ça / je laisse le travail de L et puis on pourra rajouter tous les mots que vous venez de me dire

Ici, l'enseignante, lors d'une phase de travail en sous-groupe, a travaillé individuellement avec l'élève L. Lors du regroupement, elle demande à L de 
présenter son travail, qui consiste en la création d'un écrit, concernant toute la classe, qui va servir à rappeler le rôle que chaque élève doit tenir à son entrée dans la salle de classe. Là encore, il est relativement complexe de dissocier la manière dont le travail d'écriture et l'explicitation du rôle d'élève sont pris en charge dans l'interaction. Cette complexité est d'autant plus forte, il nous semble, que l'enseignante fait également créer, par l'élève, un objet scolaire spécifique : nous aborderons cette dimension dans la partie 5 de l'article.

Dans l'exemple suivant, la frontière entre apprentissage des savoirs et explicitation de la situation scolaire nous semble encore plus mince :

Ex. 13 : classe 127, s1 :

M : je vous fais la majuscule pour l'instant celle que nous nous allons écrire/la majuscule anglaise je vais vous la faire juste pour le plaisir mais vous l'apprendrez seulement l'année prochaine [...] celle-ci [l'anglaise] on doit savoir la reconnaitre vous pouvez la rencontrer mais vous ne devez pas savoir l'écrire pour l'instant sauf si votre prénom commence par un «t»

Ici, l'enseignante présente un nouveau savoir (écrire la majuscule anglaise de la lettre «t»), mais il nous semble qu'elle explicite aussi certains aspects de l'organisation des apprentissages scolaires, en indiquant aux élèves comment l'élément qu'ils vont travailler s'inscrit plus largement dans une continuité d'acquisitions : ce qui peut leur permettre de dépasser l'instant présent pour prendre conscience de la cohérence de leur devenir d'élève.

\subsection{Expliciter la situation scolaire, organiser les activités scolaires}

L'enseignante de la classe 23 fait une référence régulière aux rôles d'élève et de «maitresse». Or l'objectif de ces références nous semble parfois ambigu, comme en témoigne l'exemple suivant :

Ex.13 : classe 23, s1 :

$\mathrm{M}:[\ldots]$ bon /gardez-les [les mots à chercher] dans votre tête/il faut d'abord que je fasse mon travail de maitresse avec le cahier jaune / [...] bon/le cahier jaune ouvert à la bonne page/pendant que je fais mon travail de maitresse j'aimerais/que / A aille faire le travail de la date sur la file/que Y fasse le travail de la date au tableau / et C/la date là-bas / on aide nos copains / [...] pendant ce temps avec votre doigt vous me montrez sur le cahier jaune où est-ce qu'on colle les nouveaux devoirs

Ici, l'enseignante est-elle en train d'expliciter une nouvelle fois qu'elle est dans un rôle professionnel, d'en préciser une des tâches, ou se sert-elle de cette connaissance pour expliciter l'organisation de la classe, en distribuant les tâches?

De manière différente, on retrouve l'ambigüité entre organisation et explicitation de la situation scolaire dans l'exemple suivant, qui concerne l'autre enseignante :

Ex. 14 : classe 127, s1 :

$\mathrm{M}$ : vous rangez les ardoises et on reprendra notre travail dans le cahier-livre sur les voyelles et les consonnes après les mathématiques 
Cet exemple témoigne, pour nous, d'une conduite explicitante qui cible la compréhension de la situation scolaire, et donc la prise en charge du rôle d'élève. En effet, l'enseignante explicite aux élèves à la fois le déroulement de la journée scolaire, en clôturant clairement une activité par une demande d'action (ranger les ardoises), et en annonçant les deux suivantes (mathématiques d'abord, puis reprise du travail consonnes/voyelles), et la structuration disciplinaire des savoirs, en utilisant le terme de "mathématiques». Elle assure ainsi clairement la transition entre des activités d'apprentissage de nature différente, alors que dans l'exemple suivant, cette transition n'est pas explicitée :

Ex. 15 : classe 127, s1 :

$\mathrm{M}$ : alors effacez bien vos ardoises [silence 15 secondes] on passe au problème?

Nous catégorisons donc en conduite explicitante le comportement verbal de l'enseignante dans le premier cas (ex. 14), mais pas dans le second (ex. 15).

\subsection{Expliciter le rôle d'élève, faire de la discipline}

Le recours régulier à la mention explicite du rôle d'élève que fait l'enseignante de la classe 23 est source d'une autre ambigüité. Ainsi, dans l'exemple suivant, l'enseignante est-elle en train d'expliciter à ses élèves le rôle qu'elle attend d'eux, ou se sert-elle de la connaissance qu'ils ont de ce rôle pour assurer, en termes de «discipline», le bon fonctionnement de la classe?

Ex. $16:$ cl. 23, s1 :

$\mathrm{M}$ : j'attends que vous soyez à votre place [...] / j'attends que vous redeveniez élèves / je vais regarder votre travail

Cette conduite récurrente pose encore plus problème lors de l'analyse des enregistrements des semaines 2 et 3 . Dans les exemples ci-dessous, il apparait clairement, notamment par l'intonation que l'enseignante emploie, qu'elle gère des problèmes de comportement de ses élèves :

Ex. 17 : cl. 23, s1 :

$\mathrm{M}$ : JE NE PEUX PAS faire mon travail de MAItresse/surtout le travail de lecture/avec d'autres enfants qui font du bruit et qui dérangent les lecteurs

Ex.18 : cl. 23, s1 :

$\mathrm{M}: \mathrm{J} / \mathrm{si}$ c'est pour te rendre intéressante/ça ne marche ABSolument pas J/et donc maintenant tu t'assois et tu vas te faire remarquer par une attitude d'élève de/CP / PAS de bébé

Nous tendons à voir dans ces interventions de l'enseignante la continuité de sa volonté d'explicitation de la situation scolaire. Si expliciter vise à la clarté cognitive chez les élèves, l'enseignant n'éprouve plus le besoin d'expliciter lorsqu'il juge que les élèves comprennent et savent ce dont ils ont besoin pour réussir dans la situation scolaire, mais il peut s'appuyer sur cette connaissance pour d'autres objectifs. Les interventions de discipline que cette enseignante juge 
utiles de provoquer sont ainsi inscrites, par leurs contenus, dans son objectif de faire comprendre la situation scolaire à ses élèves.

\section{Variété des formes des conduites explicitantes}

Nous nous sommes aussi interrogés sur les formes que peuvent recouvrir les conduites explicitantes. Lors de l'enquête, les observations ont porté sur les interactions verbales entre enseignant et élèves. Il est apparu, lors de l'analyse des vidéos, qu'il était parfois difficile de séparer l'interaction verbale des dimensions non verbales de l'action enseignante en ce qui concerne l'explicitation. Nous avons ainsi été amenés à nous intéresser au rôle que peuvent jouer, dans ces conduites, les gestes, la posture, et certains objets scolaires utilisés par les enseignants.

\subsection{Expliciter par des gestes spécifiques}

Les enseignantes observées ont toutes deux recours à des gestes issus de la méthode d'apprentissage Borel-Maisonny ${ }^{12}$, comme le montrent les deux exemples ci-dessous. Le choix de cette gestuelle spécialisée nous semble clairement traduire une volonté d'explicitation.

Ex. 20 : cl. 127, s1 :

$\mathrm{M}$ : on travaille un nouveau son qu'est-ce qu'on agrandit?

EEE : les oreilles

$\mathrm{M}$ : les oreilles/je vous donne une petite phrase dans laquelle vous allez entendre très souvent le nouveau son / pour essayer de le découvrir / attention on parle d'un son/d'un bruit/le nom de la lettre on le verra plus tard / [toRty puRkwatətcty] S?

$S:[t][\ldots]$

M : où est-ce que vous mettez votre langue pour faire ce son? I?

I : en haut des dents

$\mathrm{M}$ : en haut derrière les dents et qu'est-ce qu'elle fait la langue? I?

I : elle tape

$\mathrm{M}$ : elle tape [M fait le geste Borel-Maisonny pour le son [ $\mathrm{t}$ ] plusieurs fois] le voilà le geste / [t]

On voit ici l'enseignante travailler avec une grande clarté la dimension phonique : elle indique d'abord qu'il y a besoin d'une stratégie d'écoute, puis elle distingue clairement le nouveau phonème («un son, un bruit ») d'un graphème («la lettre»), elle en fait préciser les caractéristiques phonatoires, et fournit le geste spécifique du codage Borel-Maisonny comme le dernier élément clôturant la présentation du nouveau savoir à acquérir.

12. Pour les gestes, voir <http://www.borel-maisonny.fr/>. 
Ex. $21:$ cl. 23, s1

$\mathrm{M}$ : oui $\mathrm{B} /$ comment tu as fait pour très vite le lire ce mot?

$B$ : parce que je me rappelle que le «p» et le «h» ils font $[\mathrm{f}]$

$\mathrm{M}$ : et ouais/vous vous rappelez hier?

EEE : oui

$\mathrm{M}:[\ldots] /$ là le «p» et le «h» sont mariés et ils font le [f] [M fait le geste BorelMaisonny du son [f]] et du coup ça fait [fRaz]

Cet exemple nous permet, de plus, de pointer un comportement langagier particulier qui nous semble aussi concerner la notion d'explicitation : cette enseignante a fait le choix de construire un lexique «spécialisé» spécifique à sa classe (cf. ici le terme «mariés» qui décrit l'association graphique «ph»), pour chercher à rendre plus compréhensibles les savoirs à acquérir.

\subsection{Expliciter par des postures}

Parfois, c'est la posture entière de l'enseignante qui nous semble participer d'une conduite explicitante :

Ex. 22 : cl. 23, s1 :

[M debout dans l'embrasure de la porte de la salle de classe, tournée vers le couloir]

M : je ne parle pas puisque les enfants ne m'écoutent pas/ouvrez bien vos oreilles / ce matin dans la classe il y a un monsieur qui est là pour nous regarder / il a sa caméra/nous faisons très très attention à son matériel/et après on travaille comme d'habitude/dès qu'on entre en classe nous ouvrons notre cahier jaune à la bonne page pour la lecture des devoirs

[M entre dans la salle, va au fond de la classe déposer son manteau, les élèves entrent et vont prendre leur place]

C'est le début de la matinée, au moment de l'entrée en classe. L'enseignante se positionne dans l'embrasure de la porte, bloquant ainsi l'entrée aux élèves. Ensuite, en attendant que les élèves soient rangés, elle utilise ce temps pour diffuser des informations qui relèvent de la vie de la classe, et pour indiquer déjà les premières actions qu'elle attend de ses élèves. En mettant en place cette procédure, à la fois verbale et physique, l'enseignante nous parait signifier physiquement à ses élèves la transition entre rôle d'enfant et rôle d'élève, et spécialiser la salle de classe comme lieu d'apprentissage scolaire. C'est une conduite que cette enseignante répète tout au long de l'année.

\subsection{Expliciter par des objets scolaires}

Enfin, nos repérages vidéo ont montré la nécessité d'élargir la notion d'explicitation au recours que peuvent faire les enseignants à divers objets, dont certains qu'ils créent ou font créer par les élèves, pour les aider dans leur volonté de clarté cognitive. 
Ainsi, dans l'exemple 12 cité plus haut, l'enseignante de la classe 23 fait créer une affiche qui va lui servir à rendre plus explicite le moment où ses élèves doivent assumer leur rôle d'élève.

L'enseignante de la classe 127, elle, a créé ses propres «objets explicitants» :

Ex. 23 : cl. 127, s2 :

$\mathrm{M}$ : alors on refait le «s»/ un carreau j'écris/le carreau d'à côté je laisse libre/un carreau j'écris / [...] / fermez vos «S» / M tu as tendance à ne pas les fermer / [...] / je fais un trait penché/je redescends un tout petit peu/je vais tourner jusqu'à toucher mon premier trait/fermez vos «s» / [...] alors en dessous «une rose» / c'est un mot difficile à écrire parce qu'on a un «s» qui s'accroche sur la tête du «o» / [...] / alors le «s» il va sortir un peu de l'interligne mais vraiment très peu/on ne peut pas le fermer/on ne le ferme pas parce qu'il est accroché à la lettre «o » / on l'écrit deux fois

Dans cet exemple, l'enseignante décrit les gestes graphiques nécessaires pour écrire d'abord la lettre «s», puis le mot «rose». Cette description est une conduite explicitante puisque l'enseignante détaille une procédure d'écriture. Mais ce qui est surtout remarquable dans cet exemple, c'est qu'elle explicite les gestes graphiques en se montrant aux élèves en train d'écrire sur une grande feuille, qu'elle a préparée et affichée au tableau. Cette grande feuille reproduit en très grand les carreaux que les élèves ont dans leur page de cahier, si bien qu'elle constitue pour eux un modèle, avec les caractères que l'enseignante est en train d'écrire. La présence de cette grande feuille traduit, chez l'enseignante, un souci de précision dans l'explicitation.

Si les gestes et la posture font partie intégrante des interactions langagières, et si l'affichage, au mur et au tableau, fait partie intégrante des pratiques enseignantes, les deux enseignantes observées nous semblent faire une utilisation particulière de ces ressources, ce qui justifie de prendre ces dimensions en compte pour l'analyse des conduites explicitantes dans les pratiques enseignantes.

\section{Conclusion}

Dans cet article, nous nous sommes intéressées à l'explicitation en tant qu'elle pouvait aider les élèves à conceptualiser et à mieux comprendre le fonctionnement de l'écrit, de la lecture et de l'écriture et les amener à développer leur clarté cognitive, en englobant dans cette dernière ce qui, tout en concernant le rôle d'élève, avait une fonction dans l'apprentissage du lire-écrire. Nous avons montré que le repérage de ce que nous avons appelé les conduites explicitantes était complexe à cause de l'intrication de ces dernières avec ce qui était de l'ordre de l'énoncé des savoirs et des stratégies en matière de lecture-écriture, avec l'explication, l'organisation, la discipline. Nous avons montré que l'enseignant explicitateur n'avait pas uniquement recours à la formulation linguistique, mais qu'il pouvait également utiliser une gestuelle codifiée, et créer des objets spécifiques à la classe. 
Ce travail nous conduit à quatre remarques finales. Tout d'abord, la variété des formes que peuvent prendre les conduites explicitantes relève de ce que Lahire (2001) décrit comme les conditions indispensables au développement de l'autonomie de l'élève. Il estime, en effet, que cette autonomie, compétence visée par l'école, «repose sur trois éléments, la transparence, l'objectivisation et la publicisation» (p.153) : trois modalités que ces enseignantes nous semblent clairement mettre en place par leurs conduites explicitantes.

Ensuite, la dimension longitudinale de la pratique enseignante est à prendre en compte : les conduites explicitantes visant la clarification, en vue de leur routinisation, de la situation scolaire et de ses objets ainsi que des contenus liés à l'apprentissage du lire-écrire fixés par les programmes deviennent, au fur et à mesure du déroulé de l'année scolaire, le «terrain commun» (Clark et Brennan, $1991^{13}$ ) sur lequel enseignants et élèves peuvent implicitement s'appuyer pour réaliser les apprentissages visés. La routinisation à l'œuvre dans les classes expliquerait le fait que, comme cela a été dit dans la première partie, on constate une diminution des occurrences d'explicitation, au cours de l'année, chez les enseignants de l'enquête Lire et écrire au CP. De plus, on peut penser que de nombreuses routines seraient en fait installées dès les premières semaines du $\mathrm{CP}$, semaines qui n'ont pas fait l'objet d'observation dans l'enquête.

En troisième lieu, la présence d'une conduite explicitante chez un enseignant peut relever tout autant de la perception didactique qu'il a de son rôle dans la transmission des savoirs proprement dite, que d'une perception plus globale de ce même rôle, où dimensions pédagogiques et didactiques sont confondues et intégrées. C'est ainsi que les deux enseignantes observées ont fait des choix très différents quant à leurs conduites explicitantes. L'une, celle de la classe 23, explicite de façon régulière ce qui concerne le rôle d'élève, tandis que l'autre, celle de la classe 127, focalise ses explicitations sur les savoirs et les savoir-faire liés à l'apprentissage du lire-écrire. Ceci appellerait une autre forme de catégorisation des enseignants explicitateurs, que nous n'avons pas prise en compte dans la recherche.

Enfin, ce travail nous a permis de nous interroger sur la pertinence de certaines conduites explicitantes. Puisqu'elles reposent sur une évaluation par l'enseignant des besoins des élèves, beaucoup des conduites explicitantes observées interviennent avant même que les élèves en aient manifesté le besoin explicitement : peuvent-elles alors avoir le même effet sur tous? De même, que penser des explicitations qui sont répétées de nombreuses fois, ou dont le contenu est erroné ou discutable?

13. Clark et Brennan (1991) analysent que pour réussir à collaborer sur une tâche, deux individus doivent «coordonner à la fois le contenu et les procédures de leurs actions». La coordination du contenu nécessite de «faire l'hypothèse d'un grand volume d'information partagée ou terrain commun », la coordination de la procédure demande «la mise à jour de ce terrain commun à chaque instant». Pour eux, «tout travail collectif est fondé sur ce terrain commun et son accumulation». Une partie de l'activité langagière dans les tâches vise à la construction, et/ou la négociation, de ce terrain commun (p.127-128, notre traduction). 
Ces questionnements, qui pourraient aider à comprendre pourquoi les données relevées dans l'enquête ne permettent pas de dégager un effet significatif des conduites explicitantes en elles-mêmes sur la qualité des apprentissages de lecture et d'écriture, seront au centre de nos prochaines recherches. Le travail présenté ici pointe en effet la nécessité d'élargir notre cadre d'analyse pour y intégrer des éléments qui n'ont pas été inclus dans les codages de la recherche Lire et écrire au $C P$, et de là, l'intérêt d'approfondir la définition de l'explicitation et de ses contours dans les pratiques enseignantes ordinaires.

\section{Bibliographie}

BAUTIER, É. et GOIGOUX, R. (2004). Difficultés d'apprentissage, processus de secondarisation et pratiques enseignantes : une hypothèse relationnelle. Revue française de pédagogie, 148, 89-100.

BAUTIER, É. et RAYOU, P. (2009). Les inégalités d'apprentissage. Paris : PUF.

BISHOP, M.-F. et SRAÏKI, C. (2007). La prévention des difficultés en lecture au CP : un enjeu pour la formation des maitres. Repères, 36, 169-189.

CLARK, H. H. et BRENNAN, S. E. (1991). Grounding in Communication.

Dans L. B. Resnick, J. M. Levine et S. D. Teasley (dir.), Perspectives on Socially Shared Cognition (p. 127-149). Washington : APA Books. Récupéré le 6 décembre 2016 du site de l'université de Stanford : <http://web.stanford. edu/ clark/pubs.html>.

CRINON, J., ESPINOSA, N., GREMMO, M.-J., JARLÉGAN, A., KREZA, M. et LECLAIRE-HALTÉ, A. (2015). Clarté cognitive et apprentissage du lireécrire au CP : quelles pratiques enseignantes? Pratiques, 165-166. <https:// doi.org/10.4000/pratiques.2586>.

DOWNING, J. et FIJALKOW, J. (1984). Lire et raisonner. Toulouse : Privat.

FLAVELL, J. H. (1976). Metacognitive aspects of problem solving. Dans L. B. Resnick (dir.), The Nature of Intelligence (p.231-236). Hillsdale, NJ : Lawrence Erlbaum.

GARNER, R. (1990). Children's use of strategies in reading. Dans D. Bjorklund (dir.), Children's strategies. Contemporary views of cognitive development (p. 245-268). Hillsdale, NJ : Lawrence Erlbaum.

GIASSON, J. (1999). La métacognition et la compréhension en lecture. Dans P.-A. Doudin, D. Martin et O. Albanese (dir.), Métacognition et éducation (p.211-224). Paris : L'Harmattan.

GOIGOUX, R. (dir.). (2016). Lire et écrire au CP. Étude de l'influence des pratiques d'enseignement de la lecture et de l'écriture sur la qualité des apprentissages. Récupéré le 14 décembre 2016 du site de l'Institut français de l'éducation (Ifé) : <http://ife.ens-lyon.fr/ife/recherche/lire-ecrire/rapport>. 
GOIGOUX, R., JARLÉGAN, A., et PIQUÉE, C. (2015). Évaluer l'influence des pratiques de l'enseignement du lire-écrire sur les apprentissages des élèves : enjeux et choix méthodologiques. Recherches en didactiques, 19, 33-55.

LAHIRE, B. (2001). La construction de l'«autonomie» à l'école primaire : entre savoirs et pouvoirs. Revue française de pédagogie, 135, 151-161.

LECLAIRE-HALTÉ, A. (2007). Un impensé didactique : la mise en espace de l'exercice de lecture au CP. Repères, 36, 101-120.

MEIRIEU, P. (n. d.). Pédagogie et innovation : l'école, ses finalités et ses acteurs. Texte de synthèse. Récupéré le 14 novembre 2016 du site de l'auteur : $<$ https://www.meirieu.com/ARTICLES/pedagogie_innovation_finalites. pdf $>$

PERRENOUD, P. (1994). Métier d'élève et sens du travail. Paris : ESF Éditions.

PERRENOUD, P. (1996). Métier d'élève : comment ne pas glisser de l'analyse à la prescription? UNAPEC, Le métier d'élève. Paris : UNAPEC, 15-24.

Récupéré le 14 décembre 2016 du site de l'université de Genève, section de l'auteur : <http://www.unige.ch/fapse/SSE/teachers/perrenoud/php_ main/php_1996/1996_15.html>

PRESSLEY, M., FOREST-PRESSLEY, D. L., ELLIOT-FAUST, D. et MILLER, G. (1985). Children's use of cognitive strategies, How to teach strategies, and what to do if they can't be taught. Dans M. Pressley et C. Brainerd (dir.), Cognitive learning and memory in children (p. 1-47). New York, NY : Springer.

PRESSLEY, M. et HARRIS, K. (2008). Cognitive strategies instruction: from basic research to classroom instruction. The Journal of Education, 189(1/2), 77-94.

ROCHEX, J.-Y. (2011). La fabrication de l'inégalité scolaire : une approche bernsteinienne. Dans J.-Y. Rochex et J. Crinon (dir.), La construction des inégalités scolaires. Au cour des pratiques et des dispositifs d'enseignement (p.173-198). Rennes : PUR.

ROMAINVILLE, M. (1993). Savoir parler de ses méthodes. Métacognition et performance à l'université. Bruxelles : De Boeck Université.

ZAKHARTCHOUK, J.-M. (2000). Les consignes au cœur de la classe : geste pédagogique et geste didactique. Repères, 22, 61-81.

ZERBATO-POUDOU, M.-T. (2001). Spécificités de la consigne à l'école maternelle et définition de la tâche. Pratiques, 111-112, 115-129. 